\title{
BOOK RECEIVED
}

Chiarelli A. B., Capanna E. (Eds). Cytotaxonomy and Vertebrate Evolution. Academic Press, London. $760 \mathrm{pp}+\mathrm{XVIII}$, Price \$ 31

This book is dedicated to Professor R. Matthey, who a quarter of a century ago put into effect the idea of writing a similar work (Les chromosomes des Vertébrés -1949) based on far less abundant material. After reading the present book it becomes clear that many of the hypotheses presented in R. Matthey's work have been confirmed as correct by studies carried out during the last twenty years.

The book is divided into 5 main parts: (1) Introduction, (2) The physical basis of heredity, (3) Chromosomes dynamic, (4) Phylogenetic and taxonomic interpretation of the karyological data, (5) The sex chromosomes and the sex determination. The whole consists of 18 sections written by outstanding specialists, and of this number 4 sections are directly concerned with mammals. These are: "Chromosomal rearrangements in mammalian population polymorphism and speciation « (M. J. D. White),, Common patterns of chromosome evolution in mammalian cell cultures or malignant tumors and mammalian speciation" (L. Koulischer), "The chromosomes of non-eutherian mammals (G. B. Sharman) and "The chromosomes formulae of eutherian mammals" (R. Matthey).

The editors' intention, as shown by the title of the book, was to present complete data on the chromosomes of vertebrates, a synthesis and the most comprehensive possible evolutional commentary. In the systematic part of the book, however, certain sections (e.g. dealing with the chromosomes of fish) contain only tables of data on the number of chromosomes. The section on Eutheria has been treated similarly.

The enormous karyological differentiation and variety of cytological mechanisms participating in the evolution of mammals, and also the very great number of species, render it extremely difficult to make a synthesis relating to the whole of the group, although there are already many synthetic treatments at the level of genera or even families. The section dealing with the chromosomes of lower mammals plays the part of this kind of synthesis, but in the section on the chromosomes of Eutheria only tabular lists of number of chromosomes for over 1300 species and subspecies are given, together with statistical elaboration of these data and general remarks on the subject of chromosome mutations and the directions taken by the evolution of the karyotype of mammals.

The section devoted to the chromosomes of birds has been treated completely differently. In addition to a large $2 \mathrm{~N}$ table, there are 16 tables giving the resuits of measurements of chromosomes, which in addition are doubled by idiograms. This prodigality must irritat the reader, who will be unable to find more general conclusions based on so detailed an analysis.

The section on the polymorphism of chromosomes in mammal populations deserves mention (M. J. D. White). This section contains a discussion of the two main types of mutability of chromosomes; intrapopulation polymorphism of chromosomes and cytotaxonomic differentiation between races or species. The author draws attention to the fact that in chromosome polymorphism the conditions typical of genetic polymorphism must be maintained (predominance of heterozygotes in balanced polymorphism, adaptation to niches, the role of selection, etc.).

The book ends with indexes which have not unfortunately been sufficiently carefully prepared. The layout, consisting of photograms and figures, constitutes a very necessary supplement to the text in the majority of cases. This book, despite certain slight defects, is an interesting work containing a collection of the most up-to-date information on the chromosomes of vertebrates. 